\title{
Some Algebraic Properties of Maxwell-Dirac Isomorphism
}

\author{
Vladimir Simulik \\ Institute of Electron Physics, Ukrainian Academy of Sciences, Uzhgorod, Ukraine \\ Z. Naturforsch. 49a, 1074-1076 (1994); received September 23, 1994 \\ A new derivation of the Maxwell-Dirac isomorphism is presented. A complete set of transforma- \\ tions connecting Maxwell's electrodynamics and wave mechanics is obtained.
}

The problem of the formal connection between electrodynamics and wave mechanics has attracted the attention of a number of authors [1-8]. Here the new derivation of the Maxwell-Dirac isomorphism [9, 10] is revisited and some algebraic properties of its generating transformations are considered.

Theorem 1. Maxwell's equations of source-free electrodynamics

$$
\begin{array}{ll}
\operatorname{rot} \boldsymbol{E}+\frac{\mu}{c} \dot{\boldsymbol{H}}=0, & \operatorname{div} \boldsymbol{E}=0, \\
\operatorname{rot} \boldsymbol{H}-\frac{\varepsilon}{c} \dot{\boldsymbol{E}}=0, & \operatorname{div} \boldsymbol{H}=0,
\end{array}
$$

are equivalent to the Dirac-like equation

$$
\left[\gamma \cdot \nabla-\left(\begin{array}{cc}
\varepsilon \mathbf{1} & \mathbf{0} \\
\mathbf{0} & \mu \mathbf{1}
\end{array}\right) \frac{1}{c} \frac{\partial}{\partial t}\right] \Psi^{\mathrm{el}}=0,
$$

where in the usual representation

$$
\gamma=\left(\begin{array}{ll}
\mathbf{0} & \boldsymbol{\sigma} \\
\boldsymbol{\sigma} & \mathbf{0}
\end{array}\right)
$$

$\sigma$ are the well-known Pauli matrices, and $\Psi^{\mathrm{el}}$ is one of the two columns known from $[9,10]$

$$
\Psi_{1}:=\Psi_{1}^{\mathrm{el}}=\left(\begin{array}{c}
i E_{3} \\
i\left(E_{1}+i E_{2}\right) \\
H_{3} \\
H_{1}+i H_{2}
\end{array}\right) .
$$

Proof. By applying the matrix-differential operator of (2) to the column (4) we obtain a system of four equations in which the imaginary and real parts can easily be separated. Then, by requirening that the imaginary and real parts be equal to zero independently, we immediately get Maxwell's equations (1), q.e.d.

Reprint requests to Prof. V. Simulik.
Theorem 2. The number of columns which can be chosen as $\Psi^{\mathrm{el}}$ in (4), forming a complete set, is eight. They are, if the abbreviations

$$
E_{ \pm}:=E_{1} \pm i E_{2}, \quad H_{ \pm}:=H_{1} \pm i H_{2}
$$

are used,

$$
\begin{aligned}
& \Psi_{1}=\left(\begin{array}{c}
i E_{3} \\
i E_{+} \\
H_{3} \\
H_{+}
\end{array}\right), \quad \Psi_{2}=\left(\begin{array}{c}
-E_{3} \\
-E_{+} \\
i H_{3} \\
i H_{+}
\end{array}\right), \\
& \Psi_{3}=\left(\begin{array}{c}
H_{3} \\
H_{+} \\
i E_{3} \\
i E_{+}
\end{array}\right), \quad \Psi_{4}=\left(\begin{array}{c}
i H_{3} \\
i H_{+} \\
-E_{3} \\
-E_{+}
\end{array}\right) \text {, } \\
& \Psi_{5}=\left(\begin{array}{c}
-i H_{-} \\
i H_{3} \\
E_{-} \\
-E_{3}
\end{array}\right), \quad \Psi_{6}=\left(\begin{array}{c}
H_{-} \\
-H_{3} \\
i E_{-} \\
-i E_{3}
\end{array}\right) \text {, } \\
& \Psi_{7}=\left(\begin{array}{c}
E_{-} \\
-E_{3} \\
-i H_{-} \\
i H_{3}
\end{array}\right), \quad \Psi_{8}=\left(\begin{array}{c}
i E_{-} \\
-i E_{3} \\
H_{-} \\
-H_{3}
\end{array}\right),
\end{aligned}
$$

which includes column (4).

Proof. The validity and suitability of the set (5) can be proven by direct substitution of every column of this set for $\Psi^{\mathrm{el}}$ in (2). In order to prove that it is really a complete set let us recall the eight Pauli-Gursey operators [11]

$$
\tilde{q}=\left(1, i, \tilde{\gamma}^{4}, i \tilde{\gamma}^{4}, \tilde{C}, i \tilde{C}, \tilde{\gamma}^{4} \tilde{C}, i \tilde{\gamma}^{4} \tilde{C}\right) .
$$

They are presented here in the notation

$$
\tilde{\gamma}^{0}=\left(\begin{array}{rr}
1 & 0 \\
\mathbf{0} & -\mathbf{1}
\end{array}\right), \quad \tilde{\gamma}=\left(\begin{array}{rr}
\mathbf{0} & \boldsymbol{\sigma} \\
-\boldsymbol{\sigma} & \mathbf{0}
\end{array}\right), \quad \tilde{C} \Psi:=\tilde{\gamma}^{2} \Psi^{*}
$$


$\tilde{\gamma}^{4}:=i \tilde{\gamma}^{0} \tilde{\gamma}^{1} \tilde{\gamma}^{2} \tilde{\gamma}^{3}=\left(\begin{array}{ll}\mathbf{0} & \mathbf{1} \\ \mathbf{1} & \mathbf{0}\end{array}\right)$,

$\sigma_{1}=\left(\begin{array}{ll}0 & 1 \\ 1 & 0\end{array}\right), \quad \sigma_{2}=\left(\begin{array}{cc}0 & -i \\ i & 0\end{array}\right), \quad \sigma_{3}=\left(\begin{array}{rr}1 & 0 \\ 0 & -1\end{array}\right)$,

and form, as is well known, a complete set of pure matrix operators that leave the massless Dirac equation invariant. With the verification that the eight columns of (5) are obtained by applying separately every operator of (6) to $\Psi_{1}^{\text {el }}$ of (4) according to the rule

$$
\Psi_{r}:=\tilde{q}_{r} \Psi_{1}^{\mathrm{el}} \quad \text { for } \quad r=1,2,3,4,5,6,7,8,
$$

we complete the main part of the proof. That the columns (5) form a complete set is a consequence of the fact that the operators (6) exhaust a complete set of pure matrix symmetry operators of the massless Dirac equation, q.e.d.

There is even a kind of redundancy, in that the columns as well as the operators are pairwise interconnected by a factor of $i$ or $-i$. For example $\Psi_{2}=i \Psi_{1}$ and $\Psi_{7}=-i \Psi_{8}$. Furthermore we note the example that $\Psi_{1}^{\text {el }}$ of (4) and $\Psi_{8}$ in (5) are connected by the operator $i \tilde{\gamma}^{4} \tilde{C}$ of (6) according to $\Psi_{8}=i \tilde{\gamma}^{4} \tilde{C} \Psi_{1}^{\mathrm{el}}$.

Let us underline that the four columns $\Psi_{3-6}$ can be chosen in Theorem 2 (formula (5)) only together with simultaneous interchange $\varepsilon \leftrightarrow \mu$ in (2).

Using Theorem 1 we can complete the new derivation of the isomorphism between Maxwell's electrodynamics and the Dirac theory. Assuming harmonic time dependence in the form

$\Psi=\psi e^{-i \omega t}$ so that $\frac{\partial}{\partial(c t)} \Psi=-i \frac{\omega}{c} \Psi$,

from (2) the electromagnetic amplitude equation

$$
\left[\gamma \cdot \nabla+i \frac{\omega}{c}\left(\begin{array}{cc}
\varepsilon \mathbf{1} & \mathbf{0} \\
\mathbf{0} & \mu \mathbf{1}
\end{array}\right)\right] \psi^{\mathrm{el}}=0,
$$

follows. A comparison with the Dirac amplitude equation immediately shows the complete formal agreement of electrodynamics with the Dirac theory. A consequence of such a comparison are the formulae

$\varepsilon=1-\frac{\Phi-m_{0} c^{2}}{\hbar \omega}, \quad \mu=1-\frac{\Phi+m_{0} c^{2}}{\hbar \omega}$,

on the basis of which the hydrogen atom was presented in $[12,13]$ by means of $(1)$, i.e., in terms of Maxwell's electrodynamics.

Quite a few consequences of the connections given in (4) and (5) for quantum field theory were considered in $[14,15]$.

At the end of our considerations we want to note the following. Firstly, the generators (6) form a representation $\mathrm{D}\left(\frac{1}{2}, 0\right) \oplus \mathrm{D}\left(0, \frac{1}{2}\right)$ of the proper orthochronous Lorentz group $\mathrm{O}(1,3)$. The choice of constants for them which is necessary for yielding the commutation relations of the corresponding Lie algebra can be found in [15]. Secondly, taking into account the eight possibilities (5) of choosing the connection between $\Psi$ and $(\boldsymbol{E}, \boldsymbol{H})$, we can underline that from (5) there immediately follow also the eight possibilities of connection like (9) in [13]. Thus we have now the eight candidates for pairs like $\left(\Psi^{I}, \Psi^{I I}\right)$ in (9) of [13] that was essentially used in [13] for obtaining the hydrogen solutions. They are:

$\left(\Psi_{1}^{I}, \Psi_{8}^{I I}\right),\left(\Psi_{1}^{I}, \Psi_{7}^{I I}\right),\left(\Psi_{2}^{I}, \Psi_{8}^{I I}\right),\left(\Psi_{2}^{I}, \Psi_{7}^{I I}\right)$, $\left(\Psi_{4}^{I}, \Psi_{5}^{I I}\right),\left(\Psi_{4}^{I}, \Psi_{6}^{I I}\right),\left(\Psi_{3}^{I}, \Psi_{5}^{I I}\right),\left(\Psi_{3}^{I}, \Psi_{6}^{I I}\right)$.

In future investigations an arbitrary pair $\left(\Psi^{I}, \Psi^{I I}\right)$ from (12) can be chosen for the description of two interacting particles (waves), as it was done in [13] with the help of $\left(\Psi_{1}^{I}, \Psi_{8}^{I I}\right)$.

Taking into account the successful result of [13], we hope that by means of all eight possibilities of (12) the interaction of eight gluon fields can be usefully investigated in future research, i.e., the number of columns in (5) and pairs of possibilities in (12) is essentially connected to the number eight of gluons.

\section{Acknowledgement}

The author is much grateful to Prof. Hans Sallhofer for multiple discussions and essential scientific contribution to the paper.

$$
\left[\gamma \cdot \nabla+i \frac{\omega}{c}\left(\begin{array}{cc}
\left(1-\frac{\Phi-m_{0} c^{2}}{\hbar \omega}\right) & \mathbf{1} \\
\mathbf{0} & \left(1-\frac{\Phi+m_{0} c^{2}}{\hbar \omega}\right) \mathbf{1}
\end{array}\right)\right] \psi^{D}=0
$$


[1] O. Laporte and G. E. Uhlenbeck, Phys. Rev. 37, 1380 (1931).

[2] J. R. Oppenheimer, Phys. Rev. 38, 725 (1931).

[3] R. Mignani, E. Recami, and M. Baldo, Lett. Nuovo Cim. 11, 568 (1974).

[4] W. J. Archibald, Can. J. Phys. 33, 565 (1955).

[5] R. H. Good, Phys. Rev. 105, 1914 (1957).

[6] H. E. Moses, Nuovo Cim. Suppl. 7, 1 (1958).

[7] J. S. Lomont, Phys. Rev. 111, 1710 (1958).
[8] E. A. Lord, Int. J. Theor. Phys. 5, 349 (1972).

[9] H. Sallhofer, Z. Naturforsch. 41 a, 468 (1986).

[10] H. Sallhofer, Z. Naturforsch. 41 a, 1087 (1986).

[11] F. Gursey, Nuovo Cim. 7, 411 (1958).

[12] H. Sallhofer, Z. Naturforsch. 45a, 1038 (1990).

[13] H. Sallhofer, Z. Naturforsch. 45a, 1361 (1990).

[14] V. M. Simulik, Theor. Math. Phys. 87, 76 (1991).

[15] I. Y. Krivsky and V. M. Simulik, Theor. Math. Phys. 90, 388 (1992). 